\title{
Avaliação comparativa de formatos de placas e "plugs" para plantio de grama esmeralda ${ }^{(1)}$
}

\author{
LUIS EDUARDO DE SOUSA RAATS(2); PATRÍCIA DUARTE DE OLIVEIRA PAIVA(3); \\ MÁRCIA DE NAZARÉ OLIVEIRA RIBEIRO(3)
}

\begin{abstract}
RESUMO
A grama esmeralda é atualmente a mais utilizada no Brasil para formação de gramados, principalmente pelas suas características estéticas, rapidez de estabelecimento e rusticidade. Normalmente, o plantio é feito utilizando-se placas de $1,25 \times 0,40 \mathrm{~m}$, cobrindo totalmente a área. Mas, em algumas situações, com intuito de reduzir custos, pode ser sugerido o plantio em plugs ou placas espaçadas. Dessa forma, objetivou-se avaliar a formação de gramado utilizando diferentes tamanhos de placas e plugs, em espaçamentos variados. Assim, em um terreno preparado e nivelado, foram plantadas placas de grama esmeralda com dimensões 0,20 × 0,$25 ; 0,40$ x 0,$25 ; 0,05$ x 1,25 e 0,10 x 1,25 m em espaçamentos de 0,$10 ; 0,20$ ou $0,40 \mathrm{~m}$, além do uso de plugs nos espaçamentos 0,20 e $0,40 \mathrm{~m}$. A cada 30 dias, foram feitas medições da área, verificando o percentual de ocupação. A análise de resultados indicou que placas com formatos $0,40 \times 0,25 \mathrm{~m}$ e $0,20 \times 0,25 \mathrm{~m}$, com alta relação largura/comprimento, e espaçamento de $0,10 \mathrm{~m}$ apresentam uma taxa de ocupação da área maior, 75,89\% e 62,07\%, respectivamente. O uso de placas de dimensões $0,40 \times 0,25 \mathrm{~m}$ em espaçamento de $0,10 \mathrm{~m}$ proporcionou um redução de $36,5 \%$ no custo de implantação do gramado.

Palavras-chave: Zoysia japonica, implantação de gramados, jardins.
\end{abstract}

\section{ABSTRACT \\ Comparative analysis grass carpets sizes and plugs for zoysiagrass cultivation}

The zoysiagrass is currently the most used in Brazil for the formation of lawns, mainly due to their aesthetic characteristics and hardness. Planting is usually done using carpets with $1.25 \times 0.40 \mathrm{~m}$, covering all the area. But in some situations, aiming to reduce costs, may be suggested planting plugs or carpets with spaces of each others. This work aimed to evaluate the lawn formation using different formats of carpets and plugs in various planting distances. Thus, the land in a ground level were prepared and planted with zoysiagrass carpets with dimensions of $0.20 \times 0.25,0.40 \times 0.25,0.05$ and $0.10 \times 1.25 \times 1.25$ $\mathrm{m}$ spacing $0.10,0.20$ or $0.40 \mathrm{~m}$. beyond the use of plugs at spaced 0.20 and $0.40 \mathrm{~m}$. Every 30 days there were performed measurements of the area and observed the percentage of occupancy with zoysiagrass. The analysis results indicated that plaques formats that approximate to a square, $0.40 \times 0.20 \times 0.25 \mathrm{~m}$, with high width / length, and spacing of $0.10 \mathrm{~m}$, have an occupancy rate of the larger area, $75.89 \%$ and $62.07 \%$ respectively compared with carpets with rectangular shapes, and also higher than the planting of plugs. The use of $0,40 \times 0,25 \mathrm{~m}$ plaques, spaced of $0,10 \mathrm{~m}$, allowed an economy of $36,5 \%$ in the initial costs.

Keywords: Zoysia japonica, lawns, gardens.

\section{INTRODUÇÃO}

O gramado, como elemento paisagístico, é de fundamental importância para o embelezamento nos projetos de ajardinamento, representando, geralmente, um dos elementos principais, além de também agir como elemento de proteção do solo (LUZ et al., 2008), atuando na estabilização de encostas e no controle da erosão, entre outras finalidades (ANGELIS NETO e ANGELIS, 1999; LORENZI, 2000; RAVEN et al., 2001; FREITAS et al., 2002). Os gramados são utilizados, além da composição de jardins e conservação de solos, em campos esportivos, com destaque para os de golfe e futebol (COAN et. al., 2008; KOJOROSKI-SILVA et al., 2011; LUZ et al., 2008),

Entre os muitos benefícios dos gramados ao ambiente, destacam-se: proporcionar formação de local confortável e seguro para lazer e práticas esportivas; liberar oxigênio e manter a temperatura do ar mais confortável; reduzir a erosão do solo; purificar e absorver a água das chuvas; satisfazer o ser humano pela beleza da paisagem; além de valorizar o imóvel (TURFGRASS PRODUCERS INTERNATIONAL, 2002).

Entre as inúmeras espécies de gramíneas que ocorrem na natureza, só algumas têm aptidão para formação de gramados (SALVADOR e MINAMI, 2002), sendo produzidas em várias localidades (LANDGRAF e PAIVA, 2009). As gramíneas devem ter crescimento rápido e tolerância a cortes intensos, secas, pragas, doenças e pisoteio (LUZ et al., 2008). A escolha da espécie a ser utilizada na formação de um gramado deve seguir alguns critérios, tais como finalidade e recomendação para uso, localidade, clima, persistência, velocidade de

\footnotetext{
(1) Recebido em 17/08/2010 e aceito para publicação em 1\%11/2012.

(2) Escola Agrotécnica Federal de Machado, Rod. Machado-Paraguaçu, km 03, Santo Antonio, 37750-000, Machado-MG. E-mail: luisraats@uol.com.br

(3) Departamento de Agricultura (DAG), Universidade Federal de Lavras (UFLA), 37200-000. E-mail: patriciapaiva@dag.ufla.br; marcia_162@hotmail.com
} 
estabelecimento e qualidade estética (TURGEON, 1980), características morfológicas (KOJOROSKI-SILVA et al., 2011), sendo a grama esmeralda (Zoyzia japonica) a mais plantada (ZANON, 2003)

Atualmente, no Brasil, tem havido um aumento considerável no plantio de grama esmeralda, em função de suas características estéticas, grande resistência ao pisoteio e às variações climáticas. Mas, de modo geral, existe uma preocupação com os custos de implantação de gramados. Para minimizar, algumas alternativas têm sido sugeridas, como o plantio distanciado de placas e o uso de mudas ou "plugs". O sistema de plantio de gramados mais utilizado atualmente é o de tapetes ou placas de grama, ou sistema Maxi Rolo, em grandes áreas, que apresentam custos de implantação mais elevados em função da necessidade de mão de obra especializada e meio de transporte específico, o que encarece o frete e, consequentemente, o custo de implantação (SALVADOR e MINAMI, 2002; LUZ et al., 2008).

Assim, objetivou-se avaliar o desenvolvimento e cobertura de um terreno utilizando diferentes tamanhos de placas e "plugs", plantados em espaçamentos variados.

\section{MATERIAL E MÉTODOS}

O experimento foi realizado no município de MachadoMG, com altitude de $899 \mathrm{~m}$, clima CWA pela classificação de Koppen. A temperatura média anual é de $21,2{ }^{\circ} \mathrm{C}$ e o índice pluviométrico anual é de $1824 \mathrm{~mm}$.

A área de implantação do experimento era plana e foi preparada fazendo-se inicialmente limpeza por meio de capina e, em seguida, descompactação do solo até uma profundidade de $20 \mathrm{~cm}$. Em seguida, foi feito o nivelamento da área. Foi feita análise do solo e, pelos resultados, não houve necessidade de correção do $\mathrm{pH}$. $\mathrm{O}$ solo foi classificado como de textura argilosa, com baixos índices de matéria orgânica, ausência de Al3+ e baixos índices de P. Foi feita adubação de plantio, seguindo informações da análise de solo, baseando-se nas recomendações da Comissão de Fertilidade do Solo (RIBEIRO, GUIMARÃES e ALVARES, 1999). A cobertura foi feita 60 após dias e repetida a cada 30 dias, aplicando-se sulfato de amônio $\left(60 \mathrm{~g} / \mathrm{m}^{2}\right)$ e cloreto de potássio $(60 \mathrm{~g} / \mathrm{m} 2)$, segundo também RIBEIRO, GUIMARÃES e ALVARES (1999).

Para a instalação do experimento, as placas foram subdivididas em formatos geométricas, de diferentes dimensões, e plantadas em diferentes espaçamentos. As placas de grama foram cortadas nas dimensões de 0,20 x $0,25 \mathrm{~m}, 0,40 \times 0,25 \mathrm{~m}, 0,05 \times 1,25 \mathrm{~m}$ e $0,10 \times 1,25 \mathrm{~m}$ e plantadas nos espaçamentos de 0,$10 ; 0,20$ e $0,40 \mathrm{~m}$, tanto longitudinal quanto transversalmente em todas as combinações. Além dessas placas, também foram utilizados "plugs", plantados com espaçamentos de 0,20 ou $0,40 \mathrm{~m}$. Dessa forma, foram totalizados 14 tratamentos, implantados em 4 repetições, em delineamento de blocos casualizados e parcela subdividida no tempo. Para a uniformização dos espaçamentos, foi utilizado gabarito de madeira.

Após o plantio, as placas foram pressionadas para aumentar sua aderência ao solo e foi feita cobertura das placas e dos espaços entre elas e os "plugs" com areia grossa de rio. Para a irrigação, foram utilizados microarpersores semiautomatizados, suspensos sobre o plantio, sendo acionados em função das informações de precipitação fornecidas pela estação meteorológica do Instituto Nacional de Pesquisas Espaciais-Inpe, localizada a $200 \mathrm{~m}$ do experimento (dados fornecidos por meio dos arquivos da Escola Agrotécnica Federal de Machado). Também foram coletadas informações das temperaturas médias mensais da área, também fornecidas por essa estação.

Foram feitas medições da área ocupada por novas brotações nos espaços entre placas a cada 30 dias, até 180 dias após o plantio, com o objetivo de avaliar o percentual de ocupação da área. Para a determinação desse parâmetro, foi idealizado um gabarito de plástico transparente com divisões de $1,0 \mathrm{~cm}^{2}$. O gabarito foi colocado no intervalo entre as placas e então se procedeu às contagens de células ocupadas pelo crescimento da grama. Com essas informações, os dados foram confrontados com a área inicial e calculada a taxa de ocupação do período (área ocupada inicialmente acrescida da área ocupada no período, subtraindo-se essa soma da área total do experimento).

Os dados foram analisados por meio do aplicativo SISVAR (FERREIRA, 2011), utilizando-se o teste ScottKnott a $5 \%$ de probabilidade.

\section{RESULTADOS E DISCUSSÃO}

As dimensões das placas de grama e os espaçamentos testados influenciaram a cobertura do terreno, conforme se observa na Tabela 1. Placas com uma maior relação entre largura e comprimento e maior área apresentaram maior taxa de cobertura em relação àquelas com relação largura/ comprimento menor e, ainda, menor área em relação às mudas tipo "plug". Resultado semelhante foi obtido por NURISYAH et al. (1994), que, trabalhando com Zoysia matrella, também não observaram diferença na formação do gramado, utilizando placas com menor área.

Os espaçamentos testados influenciaram a taxa de ocupação da área. Placas plantadas com espaçamento de $0,10 \mathrm{~m}$ apresentaram melhor taxa de ocupação.

O uso de placa $0,40 \times 0,25 \mathrm{~m}$ plantada no espaçamento de $0,10 \mathrm{~m}$ foi o que apresentou a melhor cobertura, determinada pela taxa de ocupação, após seis meses do plantio $(75,89 \%)$, como pode ser observado na Tabela 1 . Já as menores taxas de ocupação foram observadas quando foram utilizados "plugs", tanto no espaçamento de 0,20 $\mathrm{m}$ ou $0,40 \mathrm{~m}$. Isso pode ser justificado pelas observações de HUDDLESTON e YOUNG (2004), que sugerem que quanto menor o espaçamento entre "plugs", maior a concorrência por nutrientes e água, o que retarda o fechamento do terreno. Nesse caso, o plantio de "plugs" espaçados de $0,40 \mathrm{~m}$ proporcionou uma taxa de fechamento inferior à observada com o plantio em espaçamento de $0,20 \mathrm{~m}$, contradizendo esses autores.

Autilização de placas com dimensões 0,40 x $0,25 \mathrm{me} 0,10$ x 1,25 m nos espaçamentos 0,40 e $0,10 \mathrm{~m}$, respectivamente, proporcionou taxa de ocupação semelhante. No entanto, operacionalmente, o preparo de placas com dimensões $0,40 \times 0,25 \mathrm{~m}$ é mais prático e rápido. Ao contrário, a preparação de placas estreitas é minuciosa e requer maior tempo e habilidade. Ainda, formatos que se aproximam de quadrado proporcionaram maior facilidade de manuseio, enquanto placas muito estreitas tiveram o manuseio mais 
dificultado, tendo ocorrido perdas de material durante os cortes e também taxa de ocupação (Tabela 1) inferior em relação às demais.

Pelos resultados apresentado na Tabela 1, é possível observar ainda que placas estreitas, com apenas $0,05 \mathrm{~m}$, tiveram desenvolvimento limitado, não proporcionando bons resultados, independentemente do espaçamento de plantio. Um pequeno incremento nessa largura, passando para $0,10 \mathrm{~m}$, aumentou a taxa de ocupação em todos os espaçamentos testados.

Analisando o crescimento em relação ao tempo de plantio, observa-se, na Figura 1, que ele foi lento entre o $1^{\circ}$ e o $3^{\circ}$ mês, tornando-se mais expressivo a partir do $4^{\circ}$ mês.

Essa observação pode ser atribuída ao fato de que, inicialmente, ocorre desenvolvimento do sistema radicular e, só então, inicia-se o crescimento da parte aérea da planta. Também se pode atribuir esse crescimento ao efeito da adubação de cobertura feita após 60 dias ou ao efeito da temperatura, uma vez que, conforme se observa na Figura 2, o período de temperaturas mais baixas ocorreu no meses iniciais de plantio.

Foi observada uma relação entre a taxa de ocupação e o tamanho da placa, sendo que placas com maior largura apresentaram maiores taxas de ocupação em relação a placas de largura menor e mesmo comprimento, conforme se visualiza na Figura 3.

O plantio de "plugs" é rápido, mas apresenta problemas de sua fixação ao solo, ocasionando desprendimento e necessidade de sua recolocação ao solo. Como vantagem, tem-se o baixo custo de implantação, que pode ser reduzido em até $50 \%$ em relação ao plantio convencional.

Analisando a economia decorrente da utilização de placas com cortes diferenciados, observa-se, na Tabela 2, que o plantio de "plugs" espaçados de $20 \mathrm{~cm}$ proporcionou baixa taxa de ocupação de área e alto custo de aquisição, em função da grande quantidade necessária de "plugs" por hectare, sendo esse custo apenas 7,14\% menor em relação ao plantio convencional utilizando tapetes. O plantio em espaçamento de $0,40 \mathrm{~m}$ reduz o custo inicial de plantio, confirmando as informações de LUZ et al. (2008). O plantio de placas com dimensões $0,40 \times 0,25 \times 0,10 \mathrm{~m}$ proporcionou melhor taxa de ocupação.

Menor custo foi calculado para o uso de placas nas dimensões $0,05 \times 1,25 \mathrm{~m}$, com espaçamento de $0,40 \mathrm{~m}$, que, apesar de ter um desempenho pouco expressivo na taxa de ocupação, foi superior ao uso de "plugs". No entanto, essas dimensões apresentam limitações (dificuldade de preparo, taxa de ocupação). $\mathrm{O}$ uso de plantas com dimensões 0,40 x 0,25 , além de proporcionar bons resultados técnicos em relação à implantação do gramado, também permite uma boa economia em relação ao cultivo tradicional, variando de 36,5 a $62,96 \%$ em função dos espaçamentos utilizados.

\section{CONCLUSÃO}

Para uma rápida ocupação de área e redução de custos, sugere-se o uso de placas de dimensões 0,40 x $0,25 \mathrm{~m}$ em espaçamento de $0,10 \mathrm{~m}$, que proporcionam edução de $36,5 \%$ no custo de implantação do gramado.

\section{REFERÊNCIAS}

ANGELIS NETO, G.; ANGELIS, B.L.D. Plantas ornamentais: do paisagismo a outras aplicações. Revista Brasileira de Horticultura Ornamental, Campinas, v.5, n.1, p.12-19, 1999.

COAN, R.M. et al. Salinidade na emergência de plântulas de duas espécies de gramas ornamentais. Revista de Biologia e Ciências da Terra, v.8, p.86-92, 2008.

FERREIRA, D. F. Sisvar: a computer statistical analysis system. Ciência e Agrotecnologia, Lavras, v.35, n.6, p.1039-1042, 2011.

FREITAS, F.C.L. et al. Efeitos do trinexapac-ethyl sobre o crescimento e florescimento da grama-batatais. Planta Daninha, Rio de Janeiro, v.20, n.3, p.477-486, 2002.

HUDDLESTON, R.T.; YOUNG, T.P. Spacing and competition between planted grass "plugs" and preexisting perennial grasses in a restoration site in Oregon. Restoration Ecology, v.12, n.4, p.546-551, 2004.

KOJOROSKI-SILVA, C.M.; et al. Desenvolvimento morfológico das gramas Esmeralda, São Carlos e Tifton 419. Ciência e Agrotecnologia, v.35, n.3, p.471-477. 2011.

LANDGRAF, P.R.C.; PAIVA, P.D.O. Produção de mudas para jardim no estado de Minas Gerais. Ciência e Agrotecnologia, v.33, n.1, 127-131p., 2009.

LORENZI, H. Plantas daninhas do Brasil: terrestres, aquáticas, parasitas e tóxicas. 3.ed. Nova Odessa: Plantarum, 2000. 349 p.

LUZ, P.B.; LANDGRAF, P.R.C.; PAIVA, P.D.O. Implantação e manutenção de gramados. In: PAIVA, P.D.O. Paisagismo: conceitos e aplicações. Lavras: Editora UFLA, 2008, 585-603 p.

NURISYAH, S.; MATTJIK, N.A.; WULANSARI, W. Effect of population density and sod size of Zoysia matrella (L.) Merr. on growth and development. Buletin Agronomi, v.22, n.2, p.16-23, 1994.

RAVEN, P.H.; EVERT, R.F.; EICHHORN, S.E. Biologia vegetal. 6.ed. Rio de Janeiro: Guanabara Koogan, 2001, p.237.

RIBEIRO,A.C.; GUIMARÃES, P.T.G.; ALVARES V.V.H. Recomendações para o uso de corretivos e fertilizantes em Minas Gerais - 5a Aproximação. Viçosa, 1999, 359p.

SALVADOR, E.D.; MINAMI, K. Avaliação de diferentes substratos no cultivo de grama esmeralda (Zoysia japonica Steud.) em bandejas. Ciência e Agrotecnologia, Lavras, v.26, n.2, p.237-243, 2002. 
TURFGRASS PRODUCERS INTERNATIONAL. Turfgrass - functional, recreational e aesthetic. Turf Resource Center, 2002. Disponível em: <http//www. turgrasssod.org/trc/statistics.html>. Acesso em maio de 2010 .
TURGEON, A.J. Turfgrass management. Virginia Reston: Reston Publishing Company, p.68-69, 1980.

ZANON, M.E. O mercado de gramas no Brasil, cadeia produtiva, situação e perspectiva. In: SIMPÓSIO SOBRE GRAMADOS, 1., 2003, Botucatu. Anais... Botucatu: Unesp, 2003. CD-ROM.

Tabela 1. Taxa de ocupação de terreno plantado com grama esmeralda em diferentes tamanhos de placas e espaçamentos após seis meses de plantio

Table 1. Occupancies rate of the cultivated area with zoysiagrass, using different carpets sizes and planting distances, six months after planting

\begin{tabular}{|ccc|}
\hline Dimensões da placa (m) & Espaçamento $(\mathbf{m})$ & Taxa de Ocupação (\%) \\
\hline $0,20 \times 0,25$ & 0,10 & $62,07 \mathrm{~b}$ \\
\hline $0,20 \times 0,25$ & 0,20 & $37,26 \mathrm{f}$ \\
$0,20 \times 0,25$ & 0,40 & $25,77 \mathrm{~h}$ \\
\hline $0,40 \times 0,25$ & 0,10 & $75,89 \mathrm{a}$ \\
\hline $0,40 \times 0,25$ & 0,20 & $60,51 \mathrm{c}$ \\
$0,40 \times 0,25$ & 0,40 & $43,71 \mathrm{~d}$ \\
\hline $0,05 \times 1,25$ & 0,10 & $37,71 \mathrm{f}$ \\
$0,05 \times 1,25$ & 0,20 & $24,99 \mathrm{i}$ \\
\hline $0,05 \times 1,25$ & 0,40 & $14,89 \mathrm{i}$ \\
$0,10 \times 1,25$ & 0,10 & $59,14 \mathrm{~d}$ \\
\hline $0,10 \times 1,25$ & 0,20 & $39,82 \mathrm{e}$ \\
\hline $0,10 \times 1,25$ & 0,40 & $29,11 \mathrm{~g}$ \\
\hline "Plugs" $(0,05 \times 0,05)$ & 0,20 & $6,46 \mathrm{j}$ \\
\hline "Plugs" $(0,05 \times 0,05)$ & 0,40 & $3,00 \mathrm{k}$ \\
\hline
\end{tabular}

*Médias seguidas de mesma letra na coluna não diferem entre si pelo teste de Scott-Knott a 5\% de probabilidade.

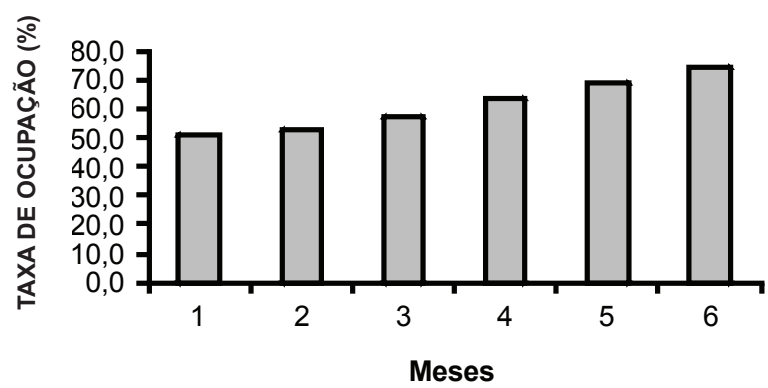

Figura 1. Taxa de ocupação(\%) da área plantada com placas de grama esmeralda de dimensões $0,40 \times 0,25$, espaçadas $0,10 \mathrm{~m}$.

Figure 1. Occupancy rate (\%) of the area cultivated with zoysiagrass carpets in sizes 0,40 x 0,25 and distance of $0,10 \mathrm{~m}$. 


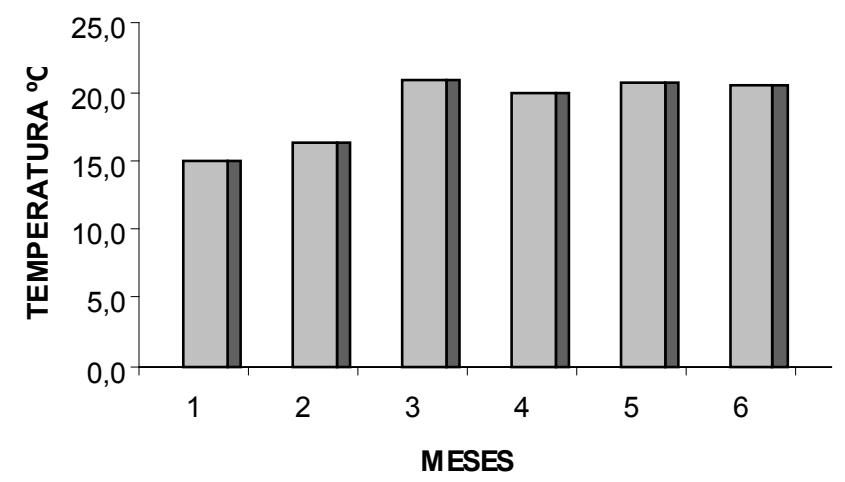

Figura 2. Temperaturas médias mensais em ${ }^{\circ} \mathrm{C}$.

Figure 2. Average monthly temperature $\left({ }^{\circ} \mathrm{C}\right)$.

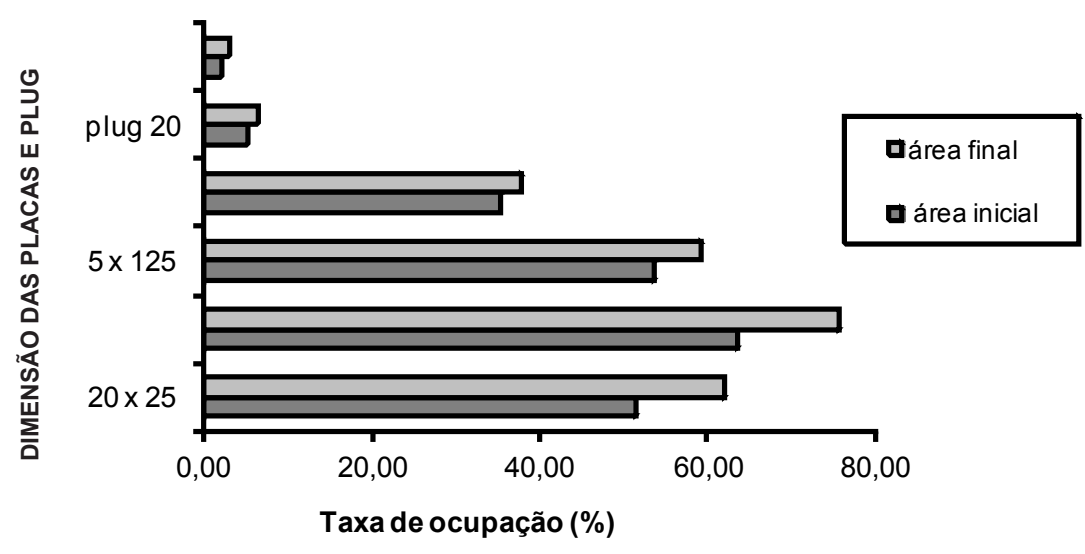

Figura 3. Taxa de ocupação (\%) por placas em diferentes tamanhos e "plugs" de grama esmeralda.

Figure 3. Occupancy rate (\%) of zoysiagrass carpets and plugs.

Tabela 2. Relação de custo de aquisição de grama em diferentes dimensões, cultivada em diferentes espaçamentos, e porcentagem de economia em relação ao plantio convencional, para a área experimental

Table 2. Relation of zoyziagrass acquisition costs in different sizes of carpets, cultivated in different distances, and economy percentages related to the conventional planting, considering the experimental area

\begin{tabular}{|c|c|c|c|c|}
\hline $\begin{array}{c}\text { Dimensões da placa } \\
\text { (m) }\end{array}$ & Espaçamento (m) & Custo (US\$*/ha) & $\begin{array}{c}\text { Ocupação Inicial } \\
(\%)\end{array}$ & Economia (\%) \\
\hline Tapetes & - & 7581,22 & 100,00 & - \\
\hline $0,20 \times 0,25$ & 0,10 & 3908,12 & 51,55 & 48,45 \\
\hline $0,20 \times 0,25$ & 0,20 & 2458,59 & 32,43 & 67,57 \\
\hline $0,20 \times 0,25$ & 0,40 & 1805,09 & 23,81 & 76,19 \\
\hline $0,40 \times 0,25$ & 0,10 & 4814,08 & 63,50 & 36,50 \\
\hline $0,40 \times 0,25$ & 0,20 & 3955,88 & 52,18 & 47,82 \\
\hline $0,40 \times 0,25$ & 0,40 & 2808,08 & 37,04 & 62,96 \\
\hline $0,05 \times 1,25$ & 0,10 & 2686,02 & 35,43 & 64,57 \\
\hline $0,05 \times 1,25$ & 0,20 & 1805,84 & 23,82 & 76,18 \\
\hline $0,05 \times 1,25$ & 0,40 & 1075,01 & 14,18 & 85,82 \\
\hline $0,10 \times 1,25$ & 0,10 & 4082,49 & 53,83 & 46,15 \\
\hline $0,10 \times 1,25$ & 0,20 & 2916,49 & 38,47 & 61,53 \\
\hline $0,10 \times 1,25$ & 0,40 & 2067,40 & 27,27 & 72,73 \\
\hline "Plugs" $(0,05$ x 0,05$)$ & 0,20 & 7039,71 & 5,33 & 07,14 \\
\hline "Plugs" $(0,05$ x 0,05) & 0,40 & 3519,85 & 2,05 & 53,57 \\
\hline
\end{tabular}

* Valor de referência $1 \mathrm{US} \$=\mathrm{R} \$ 2,77$ 